\title{
Expression of (TTF-1), CK-7 and CK-20 Immunohistochemical Marker in Neoplastic and Non-neoplastic Endometrium.
}

\author{
Sazan A. AL-Atrooshi* \\ Ameer D. Hameedi* \\ Zainab A. Zaboon*
}

\author{
FICMS \\ FICMS \\ MBChB
}

\begin{abstract}
:
Background: Thyroid transcription factor-1 (TTF-1) is a protein that is well known to be expressed immunohistochemically in normal and neoplastic thyroid and pulmonary tissues. However; increasing studies have showed its expression in normal tissues and tumors of other organs including endometrium. These facts are important to be considered in the use of this marker in the differential diagnosis of carcinoma of unknown origin and make it important to use it as a part of a panel of immunohistochemical stains. In this setting Cytokeratin 7 and 20(CK-7\&CK-20) are important since they have different coordinate expression profile in different organs and tumors.

Obejectives: This study is designed to assess expression of TTF-1, CK-7 and CK-20 in neoplastic and nonneoplastic endometrium.

Patients and Methods: Thirty six cases have been studied including 23 case of endometrial carcinoma and 13 cases of non-neoplastic endometrium including cases of irregular secretory endometrium, simple hyperplasia, complex hyperplasia, complex atypical hyperplasia, and atrophic endometrium.The study performed in the Baghdad medical city from May 2013 to December 2013

Results: Two cases out of 23 cases of endometrial carcinoma (8.7\%) showed expression of TTF-1 and none of the non-neoplastic endometrial cases showed any expression. Twenty one (91.3\%) of 23 cases of endometrial carcinoma and all non-neoplastic endometrial cases showed expression for cytokeratin 7 with different scores.Neither neoplastic nor non-neoplastic endometrium showed expression for cytokeratin 20. Conclusion:We concluded that although TTF-1 regarded as a marker for thyroid and pulmonary tissues (and tumors); other tumors "including cases of endometrial carcinomas can showed TTF-1 expression. CK7+ve/CK20-ve profile of endometrial carcinoma is important to consider in assessment of carcinoma of unknown origin especially if combined with TTF-1.

Keywords: TTF-1 CK-7 CK-20 Neoplastic non-neoplastic endometrium.
\end{abstract}

Introduction:

Hyperplastic and neoplastic endometrial disorders are common gynecological disorders and hormonal influences are important in their progression(1,2).Endometrial carcinoma is a common cancer of the female genital tract. The most common variant is the endometrioid type while other variants are relatively rare. Within the uterus and in distant metastatic sites, sometimes ; endometrial carcinoma need to be differentiated from other carcinomas that may have similar morphology. In this regard there is an immunohistochemical panel of positively and negatively staining markers for this tumor(3). This study is aimed to highlight the expression of TTF-1 in the endometrium including endometrial carcinoma and nonneoplastic endometrium.This will be done in combination with studying coordinate expression of cytokeratin 7 and 20 as these markers are important in

defining the origin of carcinoma «including endometrial» when present in an unexpected distant sites $(1,2,3,4)$.

*Dept of pathology/ collage of medicine/ University of Baghdad.

Email : ameerdhahir@yahoo.co.uk

\section{Patients and Methods:}

Thirty-six cases were studies including twenty three cases of endometrial carcinoma and thirteen cases of non neoplastic and benign endometrial lesion were selected from teaching laboratories in the medical city and from a private laboratory during the period from March 2013 and January 2014.All cases of endometrial carcinoma were hysterectomy specimen with provided information about the stage and grade of the tumor and age of the patient.Non neoplastic endometrial lesions were curettage specimens and include biopsies taken for different clinical entities and diagnoses including irregular secretory endometrium, simple hyperplasia, complex hyperplasia (with or without atypia), and biopsies for assessment of ovulation. Material:We used TTF-1 primary antibody manufactured byAbcam-USA, CK-7 from Dakocytomation- Denmark, CK-20 from Abcam-USA, and Detection Kits from both Dakocytomation and Abcam. All the following procedures of immunohistochemical staining were taken from the provided instruction with the material used (5). 
Result estimation:Immunohistochemical positivity of TTF-1 was evaluated semiquantitativly using a technique similar to that used by Siami et al et al.”(6). The average number of immunopositive cells (showed nuclear staining) within the tumor by counting 100 cells in high power field (X40) was estimated and scored using the following system Score 0 : No cell staining, Score 1: $<5 \%$ of cell staining, Score $2: 5-25 \%$ of cell staining, Score 3: $26-50 \%$ of cell staining,Score 4:51-75\% of cell staining, and Score 5: $>76 \%$ of cell staining. Alkushi et al (7) used the following scoring system for cytokeratin 7 staining (cytoplasmic staining) Negative: $<5 \%$ of cell stained, Weak to moderate positive: $5-50 \%$ of cell stained, and Strong positive: $>50 \%$ of cell stained. In our study we modulate Alkushi et alscoring system to a numerical scoring as such:Score 0 (negative) : $<5 \%$ of cell stained, Score 1 (weak to moderate positive): $5-50 \%$ of cell stained, and Score 2 (Strong positive) : $\quad>50 \%$ of cell stained. None of the cases was stained positive for Ck-20 to adopt certain scoring system for. Statistical analyses of all result were descriptive done by measuring rate, ratio, mean and standard error of mean. Results of immunohistochemical staining were analyzed using Microsoft Excel software statistical formulae.

\section{Results:}

Endometrial carcinoma:The patients age of endometrial carcinoma ranged from 38-73 years (mean 55.78). Twenty one out of 23(91.3\%) were of endometrioid type, one was adenosquamous and one was poorly differentiated . Four cases $(17.3 \%)$ were grade 1 tumor, 12 cases (52\%) were grade 2 tumors , 6(26\%) grade3 and $1(4.3 \%)$ grade 4 . TTF-1 Expression: Regarding TTF-1 expression only two cases (8.7\%) of endometrial carcinoma express this marker. One of these was of score4 (figure 1 and 2) which was a poorly differentiated tumor, the patient was the most young one of endometrial carcinoma patient in the study and the tumor did not express ck-7 nor ck-20. While the other case was of score 3 expression of TTF-1, aged 60 years and express ck-7 strongly. No significant statistical correlation was found between TTFexpression and the tumor grade, stage, or age ( $\mathrm{P}$ values $>0.05$ ) . None of the non-neoplastic cases expressed TTF-1. One case which was a case of simple hyperplasia showed cytoplasmic staining which is not regarded as a positive staining for this marker.

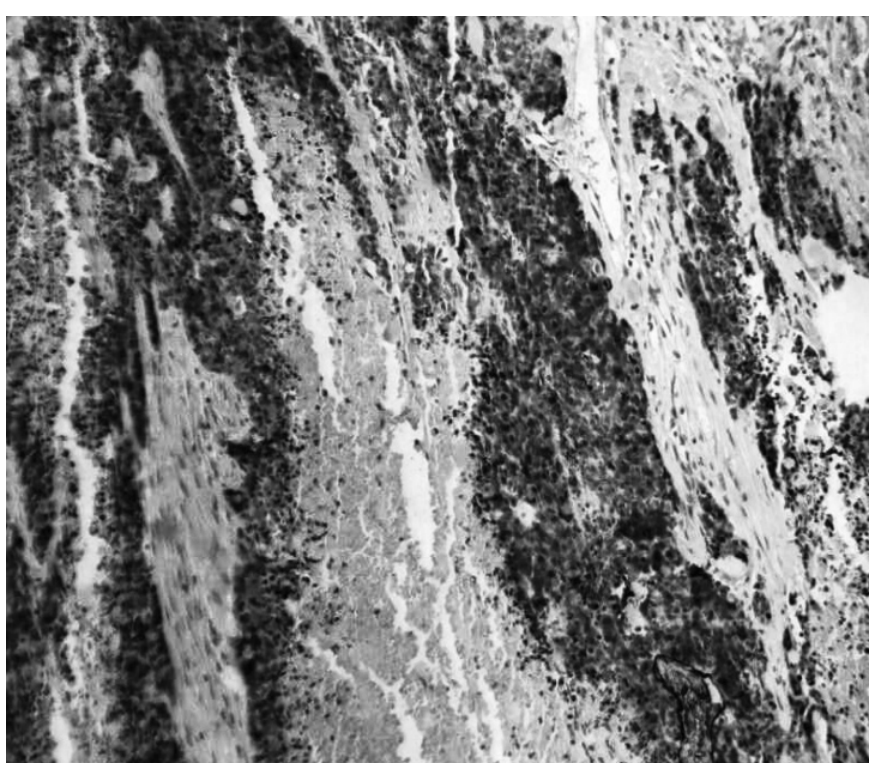

Figure 1: Score 4 staining of TTF-1 in a case of endometrial carcinoma (40X).

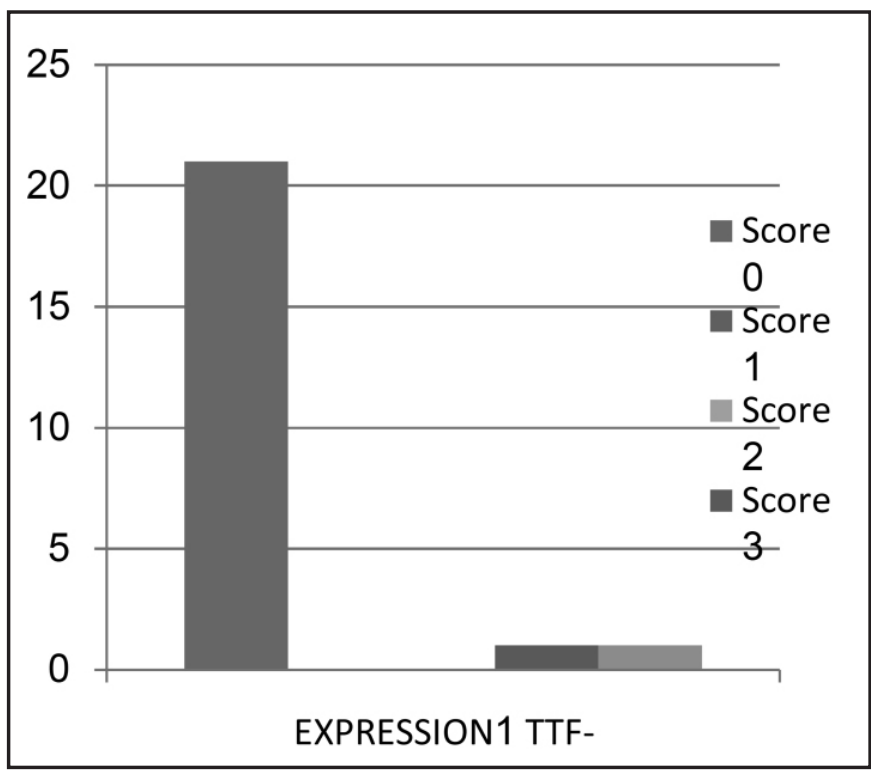

Figure 2: TTF-1Expression in cases of endometrial carcinoma which showedd that most of cases were negative.

Cytokeratin 7 and 20 expressions:For cytokeratin 7 twentyone case (91.3\%) were positive in form of diffuse cytoplasmic staining. Of them, 18(78.2\%) showed strong(score 2) expression (figure 3) and 3(13.1\%) showed weak (score 1) expression (figure 4). Two cases(8.7\%) were negative for cytokeratin 7, one was of grade 4 tumor that positively stained for TTF-1, while the other was grade 1 tumor and did not expressed any of the three markers.In adenosquamous carcinoma, the tumor showed strong expression in adenocarcinoma element while squamous element did not express the marker. 


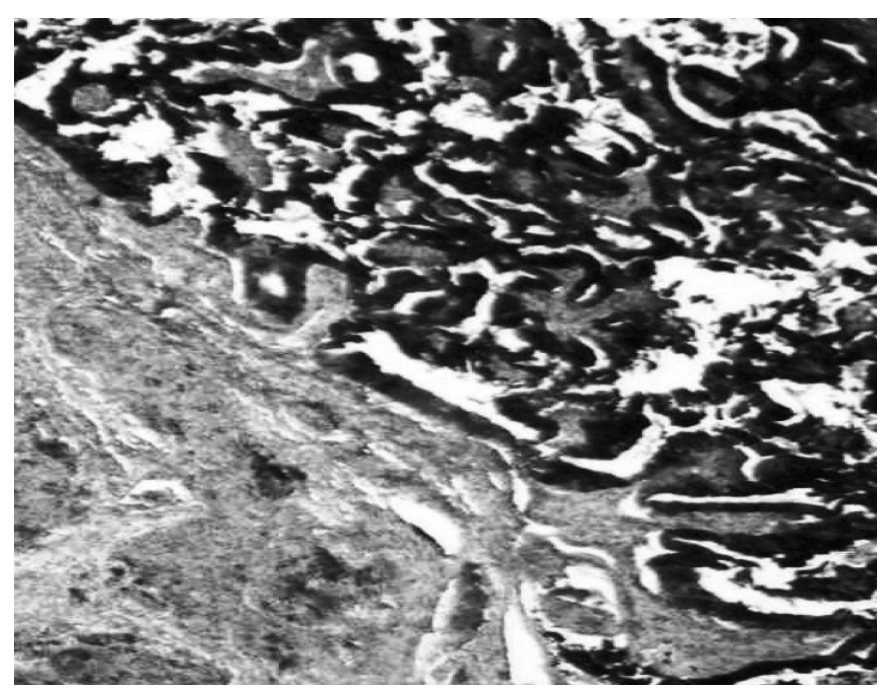

Figure 3: Score 2 (strong) expression of $\mathrm{Ck}-7$ in a case of endometrial carcinoma (10X)

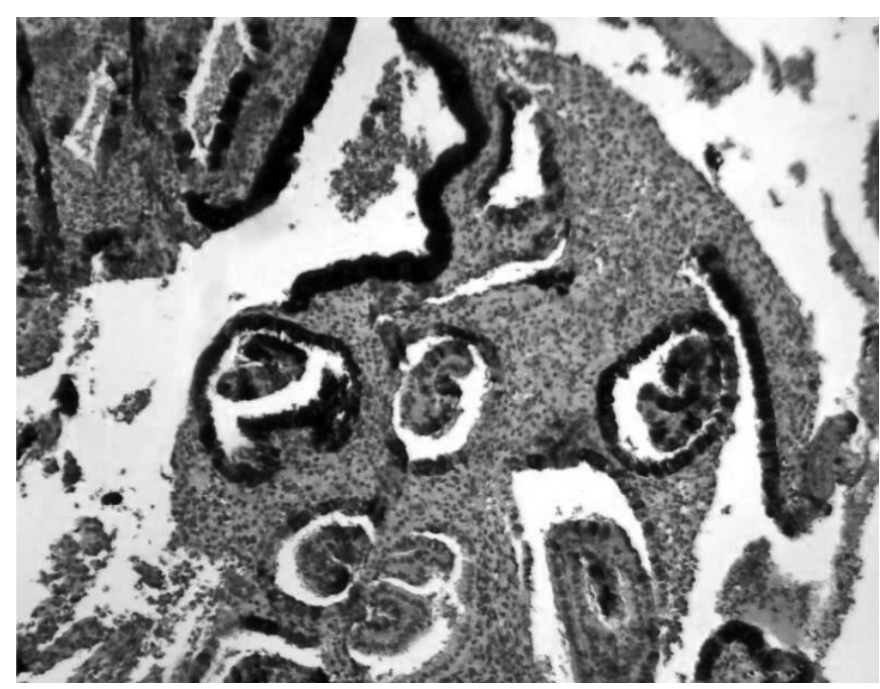

Figure 4: Score 1 (weak) expression of $\mathrm{Ck}-7$ in a cases of endometrial carcinoma (10X)

When cases of endometrial carcinoma divided into two age groups below and above 50 years, a significant statistical correlation was found between ck-7 expression score and age group (P value $<0.005$ ) (table 1 ) while no correlation was found between ck-7 expression and grade and stage of the tumor ( $\mathrm{P}$ value $>0.05$ ).

Table 1: Expression of CK-7 in endometrial carcinoma in relation to two group age distribution.

\begin{tabular}{cccc}
\hline \multirow{2}{*}{ Age } & \multicolumn{2}{c}{ No. of cases } & \multirow{2}{*}{ Total } \\
\cline { 2 - 3 } & Score 0 and 1 & Score 2 & \\
\hline$<50$ & $5(21.7 \%)$ & $1(4.34 \%)$ & $6(26.08 \%)$ \\
\hline$>50$ & 0 & $17(73.91 \%)$ & $17(73.91 \%)$ \\
\hline Total & $5(21.7 \%)$ & $18(78.26 \%)$ & $23(100 \%)$ \\
\hline P value $<0.05$ & & &
\end{tabular}

All the non-neoplastic cases (100\%) express ck-7, 3 cases (23\%) were of score 1 while 10 cases (77\%) showed score 2 expression.

None of the studied cases whether neoplastic or non-neoplastic expressed ck-20 despite repeated positive control staining. Table 2 showed the panel of co-ordinate expression of CK-7/ CK-20 for endometrial carcinoma.

Table 2: Co-ordinate CK-7/CK-20 expression in endometrial carcinoma:

\begin{tabular}{ccc}
\hline Markers & No. of cases & percentage \\
\hline CK7+ve/C20-ve & $21 / 23$ & $91.3 \%$ \\
\hline CK7-ve/CK20-ve & $2 / 23$ & $8.7 \%$ \\
\hline CK7-ve/CK20+ve & 0 & $0 \%$ \\
\hline CK7+ve/CK20+ve & 0 & $0 \%$ \\
\hline
\end{tabular}

The expression of the three markers according to age groups is explained in table 3 while the relative expression in endometrial carcinoma and non-neoplastic endometrial cases is shown in table 4

Table 3:Expression of CK7, CK20 and TTF-1 in endometrial carcinoma according to age groups

\begin{tabular}{ccccc}
\hline Age group & No. of cases & TTF-1 +ve & Ck-7 +ve & Ck-20+ve \\
\hline $31-40 y$ & 1 & $1(4.2 \%)$ & 0 & 0 \\
\hline $41-50$ & 5 & 0 & $7(30.4 \%)$ & 0 \\
\hline $51-60$ & 6 & $1(4.2 \%)$ & $8(34.78 \%)$ & 0 \\
\hline $61-70$ & 10 & 0 & $5(21.73 \%)$ & 0 \\
\hline$>70$ & 2 & 0 & $1(4.2 \%)$ & 0 \\
\hline Total & 23 & 2 & $21(91.30 \%$ & 0 \\
\hline
\end{tabular}

Table 4: relative expression of TTF-1.CK-7 and CK20 in endometrial carcinoma and non-neoplastic endometrium.

\begin{tabular}{ccc}
\hline Marker & Endometrial carcinoma & Benign endometrium \\
\hline TTF-1 & $2 / 23(8.7 \%)$ & $0 / 13(0 \%)$ \\
\hline CK-7 & $21 / 23(91.3 \%)$ & $13 / 13(100 \%)$ \\
\hline CK-20 & $0 / 23(0 \%)$ & $0 / 13(0 \%)$ \\
\hline
\end{tabular}




\section{Discussion:}

Regarding endometrial carcinoma, this study nearly agreed with other Iraqi studies in the age incidence $(8,9)$. Talal L. F and Sabaa M. (8) determined age mean was 58.5 years while Saed et al and Al-Nuaimi $(9,10)$ mean was 56.4 . In this study it was 55.78. Regarding TTF-1 staining in endometrial carcinoma in this study, we did not agreed with many studies that showed negative staining in $100 \%$ of cases of endometrial carcinoma $(11,12)$. While few other studies showed higher percentages of stained cases of endometrial carcinoma than what have been found in this study (13). Peggy et al(13) showed staining in 64\% of endometrial carcinoma and Siami et al(6) showed expression in $19 \%$ of cases. While in this study the percentage was $8.7 \%$ and the two cases that expressed TTF-1 were of different stages and grade. In non-neoplastic endometrium this study did not agreed with Peggy et al (13)who found nuclear staining in $52-55 \%$ of different benign endometrial lesion. While in this study the only stained case of benign endometrium showed cytoplasmic staining which is regarded as negative staining for this marker according to most authors( 11) and the only lesion that have been studied well for cytoplasmic TTF-1 staining is the hepatocellular carcinomas(14) and in normal hepatocytes. Niu et al (15) have identified nuclear positivity in 54\% of nonneoplastic endometrial biopsies concluding that TTF-1 might have a role in development of normal endometrial and fallopian tube epithelia. According to their finding Peggy et al (13) made a correlation between TTF-1 expression and decreased risk of progression to endometrial carcinoma upon follow-up and they concluded that TTF-1 might have a tumor suppressor activity in endometrial tissue. In the same context Nawa et al(16) and Gao et al (17) have identified TTF-1 expression as a marker of decreased incidence of carcinoma development and it may be an independent prognostic factor in endometrial carcinoma.The latter conclusions cannot be met in this study as all non-neoplastic endometrial biopsies were negative for TTF-1 and the only two TTF-1 positive cases of EC were of different stages and grade. This study agreed with many other studies that TTF-1 is not exclusively a marker for thyroid and pulmonary tumor and it can be expressed by a wide range of tumors including endometrial carcinoma (18.19). This could be of value in assessing metastatic carcinoma of unknown origin in female patient especially when present in lung and the interpretations should be made with expression of other panel of markers and the most important in this setting are cytokeratin 7 and cytokeratin 20(20,21).This study showed CK-7 expression in $91.3 \%$ of cases of endometrial carcinoma. Dennis et al (22) found staining in $60 \%$ of cases, Peiguo et al (23) in $100 \%$ of cases while Wang et al (24) found staining in $80 \%$ of cases. This study showed a correlation between the patient( age and CK-7 score that most of cases(17/23) above age of 50 years were of score 2 (strong expression)(P value $<0.05$ )
This had not been shown by other studies. This may be useful in interpreting the staining results as in case of metastasis. Only two CK-7 negative endometrial carcinoma cases were noted. One showed score 4 positive staining for TTF-1, it was grade 4 tumor and stage 4 disease. While the other case was grade 3 tumors and was negative for all three studied markers. In nonneoplastic endometrium this study showed CK-7 expression in $100 \%$ of cases of endometrial biopsies in agreement with many other studies (25) regardless the pathological process and they were of different scores. In cases that showed score 1 staining (low expression) the more tortuous proliferating glands , mostly showed secretory activity located near the surface epithelium showed more intense staining than the small regular glands that may represent stratum basalis. This may be useful in assessing the function of endometrium a fact that should be assessed in a large group study of different physiological states and pathological conditions. Nisolle et (26) al had noticed a findings that may important in this regard. They found cyclical changes in the expression of cytokeratin and vimentin in euotopic endometrium. Endometrial tissue expression of cytokeratin 7 thus has a potential value in identifying ectopic endometrium in case of endometriosis. However Song IO et al (27) have noticed lower intensity of CK-7 expression in ectopic endometrium than in eutopic endometrium. In agreement with most of related studies, neither neoplastic nor non-neoplastic endometrium showed expression for CK$20(13,28,29)$, while Wang et al (24) showed positivity in $12 \%$ of cases of endometrial carcinoma. Zemer et al (30) also noted negative staining for CK-20 in both benign and malignant endometrium, but by real time polymerase chain reaction (RTPCR) they extracted CK-20 mRNA from 17 out of 18 cases of endometrial carcinoma but none of cases of benign endometrium concluding that ck-20 may be a potential biomarker for detecting endometrial carcinoma. The coordinate expression of Ck-7/CK-20 of most of studied endometrial carcinoma cases were Ck-7 +ve/CK-20 -ve. This in agreement with most of other studies (24).The latter findings make CK-7/CK-20 expression useful in differentiating endometrial carcinoma from certain malignancies including colorectal carcinoma but of limited value differentiation between gynecological malignancies or from breast carcinoma. Many studies have investigated the expression of these three markers (TTF-1, Ck-7 and Ck-20) in different carcinomas to determine its origin. In this study $86.95 \%$ of endometrial carcinoma were: Ck-7+ve/Ck-20-ve/TTF-1-ve. This expression panel has been noted in other carcinoma such as breast and ovary but of value in differentiating EC from colorectal and few other carcinomas especially if extension of a tumor to the uterus is suspected. $100 \%$ of non-neoplastic cases were: Ck-7+ve/ Ck20-ve/TTF-1-ve. 


\section{Conclusions:}

Although TTF-1 considered as a marker that define a pulmonary or thyroid origin of carcinoma, it can be expressed in other tumors including $8.7 \%$ of endometrial carcinoma in this study.Most of endometrial carcinomas were ck7+ve/ ck20-ve being useful in differentiating them from many other non-gynecological malignancies.Ck-7 might be useful in defining ectopic endometrial tissue in difficult cases to diagnose endometriosis .Ck-7 may be expressed in different intensities in different endometrial phases.Ck-20 expression is useful to exclude endometrial origin of carcinoma and to exclude presence of ectopic endometrial tissue as none of neoplastic and non-neoplastic endometrial biopsies showed any expression for this marker.

\section{Authors contributions:}

Sazan A. AL-Atrooshi: Supervise the work progress, reviewed the case-diagnoses, interpreting the results and reviewed discussion, conclusion and recommendations.

AmeerDhahirHameedi: Cases and data collection, reviewed the case diagnoses, interpreting the results, statistical analyses and reviewed discussion and references.

Zainab Abbas Zaboon: Wrote the literature review, data and cases collection, participated in the technical part of the work (immunosataining), discussion, conclusion recommendation and references.

\section{References:}

1. Marisa R Nussi, Esther Oliva : In Gynecologic pathology By Elsevier Churchill Livingstone2009 pp 233-261.

2. Iraqi cancer registry center annual reports 2010.

3. Juan Rosai. In Rosai and Ackerman Surgical Pathology ;by Mosby Elseveir 2012Capter 19.

4. Chhieng, David C, Joan F. et al Use on TTF-1, PE-10, CK-7 and CK-20 in discriminating between primary lung carcinoma and metastatic carcinoma in FNA- aspiration. Journal : Cancer ISSN: 2001 volume 93 Issue 5, p330-336. [IVSL]

5. Provided Leaflet with Material used by Abcam and DakoCytomation companies

6. Siami K, McCluggage $W G$, Ordonez NG, et al. Thyroid transcription factor-1 expression in endometrial and endocervical adenocarcinomas. Am J SurgPathol. 2007 Nov;31(11):1759-63.

7. Alkushi A, Irving J, Hsu F, et al. Immunoprofile of cervical and endometrial adenocarcinomas using a tissue microarray. Virchows Arch.2003 Mar;442(3):271-7.

8. Talal F, Sabaa M, Evaluation of immunohistochemical staining of c-Met In Emdometrialcarcinoma.The Iraqi Post graduate Medical Journal;Vol 12. No 32013 p335-341

9. Mohammed S. Saeed, Abeer H. Ahmad, Bashar AJ
.Overexpression of Her-2/neu oncogene in 51 cases of endometrialcarcinoma Jordan Medical Journal, 2013Vol 47, No 3.

10. Wahda MT Al- NuaimyImmunohistochemical detection of estrogenreceptor $\mathrm{J}$ in endometrial carcinoma Annals of the College of Medicine Mosul 2010 Volume: 36Issue: 1\&2 Pages: 130-137

11. Hecht, J. L., Pinkus, J. L., Weinstein, L. et al . The value of thyroid transcription factor-1 in cytologic preparations as a marker for metastatic adenocarcinoma of lung origin Am J ClinPathol2001;116:483-488.

12. Stenhouse, G., N. Fyfe, et al. "Thyroid transcription factor 1 in pulmonary adenocarcinoma."J ClinPathol2004 57(4): 383-7.

13. PeggyS. Sullivan M.D.,Erin L. et al. Expression of TTF-1 in normal endometrium is associated with risk of carcinoma development. Mod Pathol 2012 August 25(8):10.1038.

14. Chin-Chen Pan, PaulChih-Hsueh Chen,, Shyh-Haw Tsay, et al Cytoplasmic Immunoreactivity for Thyroid Transcription Factor-1 in Hepatocellular Carcinoma American Journal of Clinical Pathology, 2004 121,343-349

15. Niu, Hui-Lin ; Pasha, Theresa L. B.S.; Pawel, Bruce R. et al. M.D Thyroid transcription factor -1 expression in normal gynaecological epithelium and its potential significance. International journal of gynaecological pathology. July 2009volume 28- Issue 4 - pp 301-307

16. S, Nawa A, Nakanishi T, et al. Thyroid transcription factor 1 expression in ovarian carcinomas is an independent prognostic factor. Hum Pathol.2010;41:560-5.

17. Gao HG, Pasha TL, Litzky L, et al. TTF-1 expression in ovarian and uterine epithelial neoplasia and its potential significance IntJGynecolPathol.2008;28:10-18.

18. Eva Compérat1, Fan Zhang1, Cedric Perrotin2 Variable sensitivity and specificity of TTF-1 antibodies in lung metastatic adenocarcinoma of colorectal origin.Modern Pathology (2005) 18, 1371-1376.

19. Castrillon, Diego H.; Lee, Kenneth R. Distinction Between Endometrial and Endocervical Adenocarcinoma: An mmunohistochemical Study International Journal of Gynecological Pathology:January 2002 - Volume 21 - Issue 1 - pp 4-10

20. Simsir A X J Wei et al. Differential expression of cytokeratin 7 and 20 and thyroid transcription factor- 1 in brnchiloalveolar carcinoma. Am J Clin Path 121 (3)2004 : 350-7.

21. Su YC, Hsu YC, Chai CY. Role of TTF-1, CK20, and CK7 immunohistochemistry for diagnosis of primary and secondary lung adenocarcinoma. Kaohsiung J Med Sci 2006; 22:14-9

22. Dennis JL, Hvidsten TR, Wit EC, et al. Markers of adenocarcinoma characteristic of the site of origin: development of a diagnostic algorithm. Clin Cancer Res 2005; 
11:3766-72.

23. Peiguo Chu, Emerald Wu B.S. and Lawrence $M$ Weiss Cytokeratin 7 and Cytokeratin 20 Expression in Epithelial Neoplasms: A Survey of 435 Cases Mod Pathol 2000;13(9):962-972.

24. Wang $N P$,Zee $S$, Zarbo RJ, et al. Coordinate expression of cytokeratins 7 and 20 defines unique subsets of carcinomas. ApplImmunohistochem 1995; 3: 99-107

25. Dennis JL, Hvidsten TR, Wit EC, et al. Markers of adenocarcinoma characteristic of the site of origin: development of a diagnostic algorithm. Clin Cancer Res 2005; 11:3766-72.

26. Nisolle M, Casanas-Roux F, DonnezJ .Coexpression of cytokeratin and vimentin in eutopicandometrium and endometriosis. FertilSteril. 1995 Jul;64(1):69-75.

27. Song IO1, Hong SR, Huh Y, et al . Expression of vimentin and cytokeratin in eutopic and ectopic endometrium in patient with adenomyosis.Am J Reprod Immunol.1998 Jul;40(1):2631.

28. Chu, P., Wu, E., Weiss, L. M. Cytokeratin 7 and cytokeratin 20 expression in epithelial neoplasms: a survey of 435 cases. Mod Pathol 2000;13:962

29. D Landry KT Mai. MK Senterman et al. Endometrioid adenocarcinoma of the uerus with minimal deviation of invasive pattern. Histopathology: 2003. 24: 1. Pp77-82.[IVSL]

30. Zemer R, Fishman A, Bernheim J, et al Expression of ck-20 in endometrial carcinoma. GynecolOncol. 1998 Sep;70(3):410-3. 